\title{
Enhancing Data Privacy in the Internet of Things (IoT) using Edge Computing
}

\author{
Kazi Masum Sadique ${ }^{[0000-0002-9423-6270]}$, Rahim \\ Rahmani ${ }^{[0000-0001-5924-5457]}$, and Paul Johannesson [0000-0002-7416-8725] \\ Department of Computer and Systems Sciences, Stockholm University, \\ Borgarfjordsgatan 8, SE-164 07 Kista, Sweden \\ https://dsv.su.se/en/ \\ $\{$ sadique, rahim, paul\}@dsv.su.se
}

\begin{abstract}
The vast deployment of the Internet of Things (IoT) is improving human life standards every day. These IoT applications are producing a massive amount of data from the environment where it is deployed. The collected data are mostly including end-user private data or industrial data, which are transmitted over the internet to the cloud devices for storing, processing, and sharing with the connected applications. Recent IoT data privacy-related researches are mostly focused on data privacy within a particular location of the network or at a specific device, but as per our knowledge, none has pointed and listed all the places where the end-user or industrial data privacy risks exist. In this work, we have addressed both technical and management aspects to enhance the privacy of IoT data. We have identified and listed the places where IoT data privacy risks exist, followed by our proposed model for data privacy enhancement in the internet of things (IoT). A list of ten suggestions for avoiding data privacy leakage to enhance IoT data privacy enhancement is presented. The results of this work should be useful for both academic researchers and stakeholders from the industry while designing and implementing new IoT solutions for the enhancement of human society.
\end{abstract}

Keywords: Internet of Things (IoT) - Edge computing · Identity privacy $\cdot$ Location privacy Cloud computing - IoT data privacy.

\section{Introduction}

Internet of things (IoT) applications are involved in data collection and analysis from society to enhance our daily life [15]. Vast amounts of data are produced from the IoT applications, as most of these are data-driven solutions. Some of the widespread use cases of IoT are smart home, smart city, smart grid, smart office, smart healthcare, industrial internet of things, etc. [13]. All these applications are involved in handling user's private data. The process includes data collected from the end devices, data transmission over the internet, data processing at cloud environment, and representation of results in the related user interface 
or as input to another IoT system for further results or for adjustment of the environments where the data are produced $[10,17]$. IoT data privacy can be categorized as context-oriented data privacy and content-oriented data privacy [13]. The user/device location $[10,17]$ and identity [13] are the two main components of context-oriented data privacy. The content-oriented data privacy is focused on the actual data [13]. Both context and content-oriented data privacy are essential. But based on the current use cases, content-oriented data can be accessed by third-party applications for research and development. An example use case can be access to the patient's sensitive data by a third-party research institute without proper concern from the patient. Whenever data (content or context) are shared with a third party, the data owner must get informed. There is confusion about the owner of the data. If the person/organization producing the data is the owner or the organization handling the information, is the data owner? The organization storing the data owns the right to store or share the data only after getting concerns from the person or organization producing it. Sometimes data are stored at the device (sensors, actuators, user's mobile devices), sometimes stored at IoT gateways, and at the last stage at cloud storage. As the IoT data transferred over the internet via different network devices and stored at different locations within the network, the traditional data security and privacy rules are not enough for the protection of IoT data. Motivated by the above, we have performed our research on IoT data privacy risks and possible measurements that need to be taken for any organization to protect sensitive user data at different layers of the IoT paradigm. Our research questions and contributions are described below.

\subsection{Research Questions}

In this paper, the following research questions are answered:

1. What are the IoT data privacy risk domains?

2. How can we solve the IoT data privacy problem using edge computing?

\subsection{Our Contributions}

The main contributions of this paper are as follows:

- In this paper, we have discussed the different data privacy risk domains of IoT solutions.

- We have proposed a new model based on service localization and intelligent edge gateway for the enhancement of data privacy in the internet of things (IoT).

- We have identified and listed ten suggestions for IoT data privacy enhancement, which can be used as a checklist to avoid data privacy leakages.

\subsection{Road Map for Readers}

The rest of the paper is organized as follows: in section 2, we have the related works for IoT data privacy. Section 3 describes the IoT privacy risks and proposed solution, consisting of three subsections: in section 3.1, we have discussed 
IoT data privacy risks. In section 3.2 , we have presented our proposed edge computing-based privacy enhancement model. In section 3.3, we have listed ten suggestions for IoT privacy enhancement, followed by section 4, which is the conclusion and future works.

\section{Related Works}

Recently, many researchers have discussed IoT data privacy. In [6], authors have presented a context-aware software-defined model for IoT data privacy enhancement. The authors have proposed a cloud-centric solution model for smart cities. Cloud-centric models are more vulnerable to IoT security and privacy because of more user data at the cloud layer with access by different entities. An edge centric privacy enhancement model for smart cities is proposed in [5]. The authors have presented the ontological representation of different components of a typical smart city environment with flow charts for their proposed model. They have also discussed performance evolution, but the detail about the tools used is missing. In [3], IoT risks and different IoT privacy research challenges are discussed. The proposed suggestions in this work are mostly focused on access control.

Decentralized identifier-based privacy enhancement is proposed in [9]. This work is also focused on identity and access control for privacy enhancement. But only the access control is not enough for IoT privacy improvement. Another recent research has discussed edge computing-based solutions for smart cities [16]. The authors have presented their intelligent offloading method with simulation results applied for a collaborative smart city. In [4], a detailed survey on the privacy of data at the cloud computing platform is performed. The authors have discussed different privacy challenges, applied methods for data privacy, and existing data privacy-related products. In [2], authors have extensively discussed the European Commission's data privacy rules and regulations, namely the General Data Protection Regulation (GDPR). They have also discussed in detail their Snap4City smart city solution. In [8], authors have presented their work on local monitoring and evolution of network traffic from IoT devices and connected mobile devices. This work is focused on the localization of data for privacy enhancement. As per our knowledge, none of the recent research has addressed the points we have discussed in detail in this paper.

\section{IoT Privacy Risk Domains and Proposed Solution}

In this section, we have discussed different IoT privacy risks at different layers of the IoT paradigm, followed by our proposed edge-centric location-based IoT privacy enhancement model. At the end of the section, we have briefly described our identified ten privacy enhancement measurements. 


\subsection{Identified IoT Privacy Risk Domains}

IoT data privacy risks exist at different stages of data collection, data analysis, and storing processes. For enhancement of IoT data privacy, it is essential to identify each of the individual domains where there are possibilities of the potential risk of data privacy leakage. In our option, a domain can be anything like a software application, hardware, a network device, an activity performed by the user, an automated activity conducted by a network device, or a server. Based on our above definition, we have identified the following domains where IoT privacy risks need proper attention:

IoT End Devices: Third parties can possess IoT end devices, and they can exact data. The end devices are mostly unmanaged and unmonitored, allowing adversaries to take control of those devices and extract data from them. For example, if a door sensor at a personal property is hijacked, hackers can extract data from it and perform harmful activities when the proprietor is not present at the location. The same applies to medical sensors. If bad guys get control of end devices, they can extract data from it. As a result, IoT end devices have a risk of privacy leakage.

IoT Gateways: Adversaries can possess an insecure IoT gateway. IoT gateways can have similar privacy risks as the IoT end devices. But the IoT gateway holds more data than an IoT end device, so it is more vulnerable. A hijacker of an IoT gateway can extract more data about any person or organization if he/she gets access to an IoT gateway. Besides the risks of data extraction, possession of an IoT gateway has more risks if the intruder gets write access to the device and can perform many dirty activities, which may lead to loss of assets and even loss of life if it is related to healthcare.

Cloud Devices: Cloud computing is famous for storing and analyzing IoT data as it has enough storage, memory, and computational power to perform many complex tasks. Besides scalability, security, storage, and analytics, cloud computing facilitates inter-organizational communication. The cloud application programming interface (API) allows organizations to collaborate and share data. While sharing data with other organizations, it is a risk factor for the company to lose its or employee's private data due to wrong configuration and lack of proper authorization in the back-end system.

Mobile Devices: The user's mobile devices are enhanced with many capabilities to track different activities performed by the user. For example, a step count application keeps track of walking; many location-enabled applications keep track of the user's location, and so on. A mobile device has privacy risks because if it is lost or possessed by a third party, the user's data can be extracted from the device. Also, mobile devices may have few applications installed by the 
user, collecting and sending personal data to a server without taking concern from the user. A mobile device is also at risk when used as a data collection or analysis tool because access to insecure data sources can make the device vulnerable.

Automatic Data Upload: One of the features of recent mobile applications is the auto-upload of user data to the service providers' cloud storage. Though it is a useful facility and users don't need to worry about the details, it introduces data leakage risks. For example, a user's data can be sent to cloud storage and accessed by the employees within the application developer's organization, which is not acceptable if the user does not approve it on that device.

Insecure Communication Channel: Many IoT applications communicate between each other's, with the gateways, with the cloud storage over insecure communication channels, allowing man-in-middle attackers to collect data from the network and further perform harmful and malicious activities.

Databases: There are privacy risks at the backend databases. IoT data stored in databases can be extracted due to insecure configurations. For example, a web application's front end can have more data sent from the server, which is not shown at the front end of the application but can be extracted from the data variable of the source code of that front end. Also, SQL injection can be performed to extract data from poorly configured databases.

Unauthorized Access: There exist vast risks of unauthorized access to IoT data. In many cases, IoT devices and related storage devices are configured with authentication mechanisms only. While at a big organization, it is crucial to have a proper authorization mechanism over the authorization processes. For example, in a healthcare application, a nurse may not have accessed part of the patient data, and even a general physician may not be allowed to access part of the patient data without proper concern. But a poorly configured system may lead to privacy risk.

People's Awareness: People's awareness is very crucial. IoT privacy risks exist due to a lack of understanding and knowledge. An example scenario can be a mobile device user using mobile devices without a password or pin code. In this scenario, if the mobile is lost, an unauthorized person will access the user's data. Another example could be a Wi-Fi password. Without enough knowledge, general users may use Wi-Fi devices without changing the default passwords. Intruders can easily monitor the network traffic by connecting the Wi-Fi using the default pin code/password. They may access the user's private data. 


\subsection{Proposed Model}

As discussed in the previous section, there are several IoT data privacy risks at different IoT paradigm layers. To solve the issue mentioned above, we have designed a new model (see Fig. 1). In our model, IoT services and applications are localized at the edge devices and within the cloud computing layer. IoT devices within a specific location transfer data to the nearest edge gateway(s). The intelligent edge gateway only shares part of the collected data with the cloud devices. The spatiality of the edge devices is data filtering before transferring data to the cloud. The edge IoT devices are responsible for data aggregation and processing and data storage as well. In our proposed model, the end-user IoT applications communicate to the cloud servers to access data or information processed at the cloud devices. In specific scenarios, the IoT end-user applications can directly access the edge gateways. This model enhances the IoT data security and privacy by localizing services and reducing data transfer between the IoT gateways and the cloud devices.

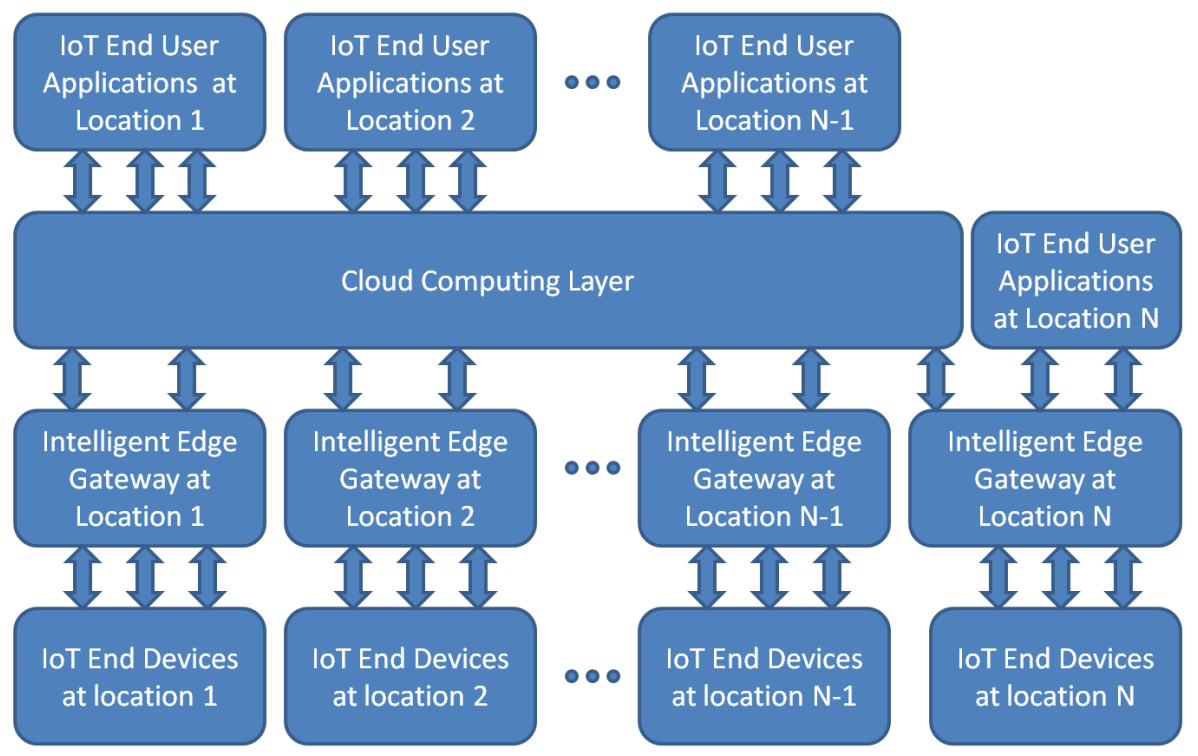

Fig. 1. Proposed IoT privacy enhancement model using edge intelligence and localization of services.

To understand our proposed model, we can consider an example scenario: a user may have access to data collected from different locations. IoT devices are connected to the IoT gateways at different locations. Access to the data is only allowed after the data filter at the intelligent edge gateway. The gateways collect the data and share part of the data with cloud storage. In some use cases, user end-device applications access the data from the edge gateways.For proper 
privacy implementation, an intelligent edge gateway verifies users' identity and authorization before allowing access to any data source.

It could happen that intruders got access to the IoT gateways. So, it is essential to have private data filtered before the intruder gains access. The intelligent IoT gateways need to identify sensitive personal information so that the private data can categorize. In such cases, intelligent gateways will use machine learning algorithms to categorize data and sources. As machine learning algorithms required more resources and the edge gateways may have limitations, so we propose Support Vector Machine (SVM) [7,14] or Naive Bayes [14] as the machine learning algorithm. But at the initial stage, the machine learning algorithm will not have enough data to predict user activity. As a result, a rule-based classifier $[11,1]$ can be used for data and source/user categorization at the initial stage. Rule based classification can be performed with very few stages/steps. [1]. Fig. 2 represents different steps for rule based categorization.

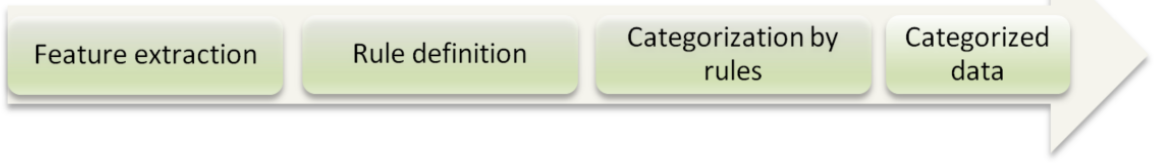

Fig. 2. Steps for rule based categorization.

The intelligent edge gateways will also include trusted storage modules to protect unauthorized access to users' private data. A trusted storage module can be build based on the concept of trusted execution environment [TEE] [12]. To restrict access to trusted storage area, source categorization can be implemented. We propose the following simple algorithm for source filtering:

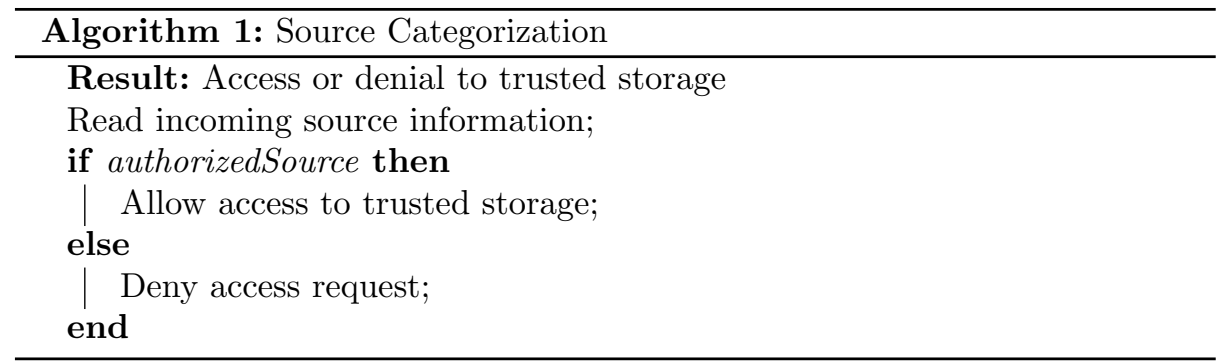

It is also important to categorize data before it is stored in the data storage. An intelligent gateway will categorize the data based on the data type. Demographic data should be stored in the storage with extra padding, and the padding values should be secret. We propose the following simple algorithm for data categorization: 


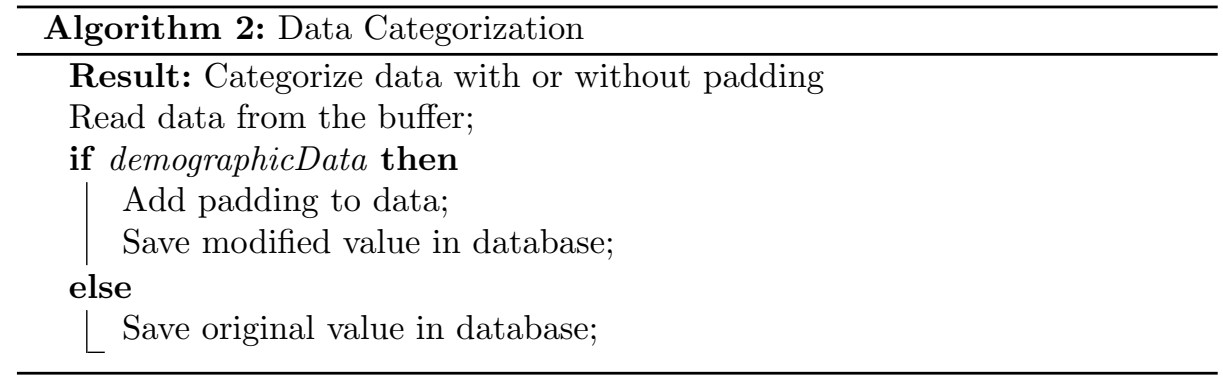

\subsection{Suggestions for improvement of IoT data privacy}

We have identified ten main points for the enchantment of IoT. Our identified points are valid for any IoT applications and give research directions for academics and industrial bodies. Detailed descriptions of our identified points are below:

Ensure Security at Devices to Enhance Privacy: IoT devices are mostly unmonitored and remotely mounted for data collection from the environment it is applied. The physical security of the device is essential for the enhancement of the privacy of the IoT data. The communication between the device and the nearest IoT device must be secure and needs to be tempered resistance. Remote connections to the IoT devices need to be restricted. In case the device allows remote connection, it should always have a strong password to protect it from hackers. For example, cameras mounted at smart buildings and assets monitoring are mounted at different locations and are mostly not physically monitored by the authorities. In case an intruder gets access to the data generated by these cameras can perform analysis of activities of any authorized person within that area and make harmful activities to him or on the property itself based on his intention.

Ensure Security during Communication to keep Data Privacy: As the data collected from the IoT devices are mostly transferred to the cloud, secure communication between the IoT end devices and the cloud servers is worthy. Due to the heterogeneous distribution of IoT devices, traditional network security cannot completely secure the IoT data during communication.

The communication security for the enhancement of IoT data privacy needs at three different stages:

- At the communication between the IoT devices and the IoT gateways

- At the communication between the IoT gateways and the cloud storage

- At the communication between the cloud servers and end-user IoT applications

Any eavesdropping on data at these three stages of communication is against the privacy of IoT data. Due to the difference in communication media and 
communication protocols, security implementations should be different at these three stages. For example, an end device can connect to the IoT gateway using wireless communication like Bluetooth, or Zigbee or Wi-Fi or mobile network (3G, 4G, and 5G), etc. An IoT gateway may have a wired connection to the cloud servers. And the cloud servers may share results to the end-user applications using wired or wireless communication based on the end-user devices.

IoT Data Privacy at IoT Gateways: IoT data is collected, aggregated from the raw sensor devices, and processed at the IoT gateways before sending it to the cloud server. Implementation of appropriate security rules at IoT gateways is significant for the privacy of data. The IoT gateways should have proper authentication and authorization mechanisms for the protection of data. The IoT gateway itself is vulnerable to security attacks. The remote communication to the IoT gateways needs to be secure and restricted. For example, a secure shell (SSH) connectivity should be allowed only from a trusted entity. Private tunnels between the IoT gateway and the service provider's devices can improve security and privacy. Here the service providers are the entities who are the owner or the maintainer of the IoT gateway. Access to the IoT gateway data needs to be restricted even for the employees within the service provider organization.

Enforcement of Cloud Layer Data Privacy: The Cloud layer provides scalability, flexibility, robustness, reliability, and more secure data storage and processing than any other layer where the IoT data is stored and processed. Besides the benefits, cloud layer data processing can increase data privacy risk because IoT data becomes more visible and accessible to the third party at the cloud layer. For example, application programming interface (API) connectivity to the cloud data is a prevalent tradition for integrating organization and the increase of productivity at different organizations. API based data access is mostly performed using authentication tokens. Sometimes the same token is used for all the communications between the requester (client device) and a cloud server, but accessing different endpoints and resources using the same access token increases the data privacy risks. There could be scenarios where the authentication tokens have to be changed periodically with the increased number of requests from the clients. In such a case, A rule based classifier model (see Fig. 2 ) can be used. The users get notified, and the token should be revoked so that the same token cannot be used again in further request. An unauthorized person within the service provider organization can read and modify the private data of end-users using the token. It is hard to track the data modifier if several persons within the organization use the same authentication token. Another privacy issue at the cloud layer is the implementation of data access policies for the users and organizations accessing the data. It is prevalent at the current age that IoT device users use his/her username and password for authenticating third-party applications to access his/her data from the cloud. But if the authorization processes at the cloud servers are not strict, the third party can get access to sensitive private data of the user, while the users didn't get informed at all of this. 
Proper Enforcement of Access Rules on Data Storage: IoT data can be stored at many different devices and accessed by various applications. The most common data storages are IoT devices, where the data is stored for a tiny amount of time before transferring to the IoT gateways. The following data storing devices are IoT gateways, at the cloud devices, and at the end-user mobile application and/or computers from where the results are accessed. Based on scenarios, databases are accessed by the IoT user's application, cloud applications for data analysis, third party applications via APIs, and IoT applications that are used for adjustment to the environment (machine to machine communications). Implementation of access rules with proper authentication is crucial for the privacy of IoT data. API access is implemented for external access to the databases from the third-party application, needs more attention. The same rules applied for the IoT gateway storage. Also, for the privacy of IoT data at gateway devices, it is necessary to monitor access to the devices. While considering data privacy at the end-user devices, it is important to store the data in a manner that other applications cannot access the data and if the device is stolen, access to the data is not possible by the thief. For example, the users may have the option to erase their personal data by login into his application from another device, which will increase user data privacy. Another important point is logging of access to the data. It is not easy to log all the activities, but the unwanted and abnormal movements need to be logged in the databases.

Role-based Authentication and Authorization Model: It is crucial to ensure proper authentication and authorization to IoT data in the cloud as the cloud data is more visible to different users, organizations, and applications. Role-based authentication and authorization can improve data privacy. In a rolebased model, users or applications get access only to the part of the data; it should need access, and other parts of the data have restricted access. The same rule is applicable for API based access to the database. The APIs should be designed very carefully with proper authentication and authorization rules to enhance data privacy.

Limited Data Sharing with the Cloud: As the cloud layer is more visible from the world, it is better to store only relevant data to the storage and limit storing private data, which is not related to the specific IoT application for which the data is collected. This increases data privacy and reduces the risk of access to the user sensitive data by third parties. For example, a user's full name, address, personal social security number, age, weight cannot be relevant for some applications, and it could be useful for other applications. In these scenarios, the data storage in the cloud should be designed so that only relevant data should be stored and accessed from the IoT gateways. Limited data sharing also reduces the risk of eavesdropping on private data while transferring via the internet.

Implementation of Edge Intelligence to Enhance Data Privacy: We have proposed a model in which the IoT data processed at the edge IoT gate- 
way and transferred to the cloud for further analysis and storage if required. Intelligent edge gateways will enforce rules on IoT data as closely as to the data source. It will filter the data before transferring it to the cloud. If required, the edge devices will make necessary changes to the data fields (e.g., a dummy value assigned to the private data) before transferring to the cloud layer. The intelligent edge devices will also quickly make decisions near the data source and share it with the related IoT applications. Real-time data processing is crucial, and less interaction with the cloud is needed. Intelligent edge gateways can also log unauthorized and abnormal activities attempted by different applications and generate alarms if any data privacy violation has occurred.

User Awareness and Awareness of Peoples Working with Data: People's awareness of data privacy is crucial. The IoT application users should get accurate information about the data they share and generate using their applications. Sometimes in different user applications, the legal agreements are so long and hard to understand by the end users that users lose interest in reading the details and press the agree on buttons before reading it. The legal documentation on any application needs to be easily understandable for the end-users and should be as short as possible. While preparing a legal document, it is essential to consider that the legal authorities' level of legal knowledge and the end-users are not the same.

Law Enforcement and Law Awareness for the Peoples Working with Data: There are laws related to data privacy that needs proper enforcement all over the world. As the data on the internet can travel the world, the laws for data privacy leakage should be at the same level worldwide, and people's awareness about it is worthy. Any employee joining an organization where personal data is processed should get proper training on the data privacy requirement and know his right to access data at different systems.

\section{Conclusion and Future Works}

In this paper, we have discussed IoT data privacy risks at different stages of an IoT paradigm. We have proposed an edge centric privacy solution for the IoT paradigm. We have also identified ten main points that need to be considered while implementing an IoT application scenario. The identified ten points can be considered as a checklist for the privacy enhancement of any IoT solutions. Our proposed model will reduce the leakage of sensitive user data during transmission and due to loosely configured cloud access. It will also protect from the entry of intruders who may have access to user data but are dishonest. We have suggested a rule-based classifier at the early stage of deployment. After a specific time, the system will have enough data for SVM or Naive Bayes machine learning algorithms to predict unauthorized malicious activities. We will extend our work on the intelligent edge gateway model for the IoT data privacy enhancement with validation in our future work. 


\section{References}

1. Arefi, H., Alizadeh, A., Ghafouri, A.: Building extraction using surface model classification (2013)

2. Badii, C., Bellini, P., Difino, A., Nesi, P.: Smart city iot platform respecting gdpr privacy and security aspects. IEEE Access 8, 23601-23623 (2020)

3. Bertino, E.: Data privacy for iot systems: Concepts, approaches, and research directions. In: 2016 IEEE International Conference on Big Data (Big Data). pp. 3645-3647. IEEE (2016)

4. Domingo-Ferrer, J., Farràs, O., Ribes-González, J., Sánchez, D.: Privacy-preserving cloud computing on sensitive data: A survey of methods, products and challenges. Computer Communications 140, 38-60 (2019)

5. Gheisari, M., Wang, G., Chen, S.: An edge computing-enhanced internet of things framework for privacy-preserving in smart city. Computers \& Electrical Engineering 81, 106504 (2020)

6. Gheisari, M., Wang, G., Khan, W.Z., Fernández-Campusano, C.: A context-aware privacy-preserving method for iot-based smart city using software defined networking. Computers \& Security 87, 101470 (2019)

7. Huang, J., Lu, J., Ling, C.X.: Comparing naive bayes, decision trees, and svm with auc and accuracy. In: Third IEEE International Conference on Data Mining. pp. 553-556. IEEE (2003)

8. Klement, F., Pöhls, H.C., Spielvogel, K.: Towards privacy-preserving local monitoring and evaluation of network traffic from iot devices and corresponding mobile phone applications. In: 2020 Global Internet of Things Summit (GIoTS). pp. 1-6. IEEE (2020)

9. Kortesniemi, Y., Lagutin, D., Elo, T., Fotiou, N.: Improving the privacy of iot with decentralised identifiers (dids). Journal of Computer Networks and Communications 2019 (2019)

10. Malina, L., Srivastava, G., Dzurenda, P., Hajny, J., Ricci, S.: A privacy-enhancing framework for internet of things services. In: International Conference on Network and System Security. pp. 77-97. Springer (2019)

11. Qin, B., Xia, Y., Prabhakar, S., Tu, Y.: A rule-based classification algorithm for uncertain data. In: 2009 IEEE 25th International Conference on Data Engineering. pp. 1633-1640. IEEE (2009)

12. Sabt, M., Achemlal, M., Bouabdallah, A.: Trusted execution environment: what it is, and what it is not. In: 2015 IEEE Trustcom/BigDataSE/ISPA. vol. 1, pp. 57-64. IEEE (2015)

13. Sarwar, K., Yongchareon, S., Yu, J.: A brief survey on iot privacy: Taxonomy, issues and future trends. In: International Conference on Service-Oriented Computing. pp. 208-219. Springer (2018)

14. Williams, N., Zander, S., Armitage, G.: A preliminary performance comparison of five machine learning algorithms for practical ip traffic flow classification. ACM SIGCOMM Computer Communication Review 36(5), 5-16 (2006)

15. Wong, K.S., Kim, M.H.: Towards self-awareness privacy protection for internet of things data collection. Journal of Applied Mathematics 2014 (2014)

16. Xu, X., Huang, Q., Yin, X., Abbasi, M., Khosravi, M.R., Qi, L.: Intelligent offloading for collaborative smart city services in edge computing. IEEE Internet of Things Journal 7(9), 7919-7927 (2020)

17. Zhou, X., Yang, L., Kang, Y.: A research on the iot perception environment security and privacy protection technology. In: International Conference on Frontier Computing. pp. 104-115. Springer (2017) 